\title{
MOVILIDAD OCUPACIONAL EN CHILE 2001-2009 ¿Desigualdad de ingresos con igualdad de oportunidades?
}

\author{
OCCUPATIONAL MOBILITY IN CHILE 2001-2009 \\ Inequality of income with equal opportunities?
}

\author{
VICENTE EsPINOZA vicente.espinoza@usach.cl \\ Universidad de Santiago. Chile.
}

JAVIER NúÑEZ jnunez@fen.uchile.cl

Universidad de Chile. Chile.

\section{REsUmen}

El diagnóstico de la sociedad chilena como "desigual pero fluida" (Torche 2005) que caracteriza la visión actual de la movilidad social fue contrastado con datos de la Encuesta Nacional de Estratificación Social (ENES) aplicada en 2009, lo cual permite apreciar la evolución de la movilidad social intergeneracional a lo largo de la década. El artículo modela las pautas de movilidad ocupacional replicando el modelo de fluidez constante de Erikson y Goldthorpe (1992), así como su adaptación a Chile por Torche. Los resultados que se presentan discrepan de los obtenidos por él pues nuestro análisis muestra mayor rigidez en el régimen de movilidad, especialmente por el peso de las barreras jerárquicas y sectoriales. Dado el peso de la jerarquía y la superposición de ésta con las barreras sectoriales se explora un modelo de movilidad "vertical", el cual proporciona un ajuste satisfactorio en ambas muestras. Finalmente el artículo explora la asociación entre la dimensión vertical y los ingresos de los grupos ocupacionales. Estos hallazgos abren una nueva agenda de investigación respecto a las oportunidades que ofrece la sociedad chilena a sus integrantes.

\section{Palabras Clave}

Desigualdad social; Movilidad de ingreso; Movilidad ocupacional.

\begin{abstract}
The paper compares the regimes of intergenerational occupational mobility in Chile between 2001 and 2009. Data for the study considers published mobility tables for 2001 as well as original data for 2009 from the National Survey of Social Stratification (ENES). The analysis of mobility patterns replicates the constant fluidity model proposed by Erikson and Goldthorpe (1992), considering also special parameters for Chile as proposed by Torche (2005). His depiction of Chilean society as "unequal but fluid" is challenged by the 2009 data in two special respects: First, we find a stronger hierarchical effect and a larger sectoral barriers to mobility than previously reported. Secondly, the latter yields a less fluid mobility regime. The paper also explores the hierarchical dimension of mobility by testing a "vertical" model (RCII), which provides an adequate fit in both samples. Finally, the paper compares the vertical dimension of occupational mobility with income distribution for occupational groups. These findings open a new agenda for the study of the opportunities that Chilean society offers to its inhabitants.
\end{abstract}

\section{KEYWORDS}

Income Mobility; Occupational Mobility; Social Inequality. 


\section{INTRODUCCIÓN*}

A lo largo de los últimos 20 años la pobreza en Chile se redujo desde niveles cercanos al $40 \%$ hasta alcanzar alrededor de $15 \%$, constituyéndose en el caso más exitoso en la región latinoamericana, merced a un encomiable esfuerzo de política social aunada con crecimiento y equilibrio macroeconómico. La superación de la pobreza constituyó el motivo y horizonte de la política social en las últimas dos décadas, objetivo que en gran medida se asociaba con la inserción en el mercado de trabajo. En este período, la política social del sector público comenzó a desplazarse primero hacia los grupos sociales con más dificultades para superar la condición de pobreza, estableciendo una focalización de alta precisión para terminar con las formas más extremas de pobreza. Posteriormente, las políticas y programas de "protección social" ampliaron el foco de la política social hacia grupos en riesgo de entrar en la pobreza, lo cual comprende alrededor del $40 \%$ de la población con menores ingresos.

Llamó la atención a los analistas del proceso que la superación de la pobreza no estuviera asociada con una modificación de los niveles de desigualdad de ingresos, pues los incrementos en el ingreso total de la población no alteraban su distribución (Contreras 2003; Joignant \& Güell 2009). En las últimas tres décadas, Chile experimentó una notable expansión económica, con un crecimiento medio de alrededor del $5 \%$ anual, alcanzando un ingreso per cápita para 2009 de dólares 14.331 (PPA), y sus autoridades esperan que en poco más de una década se encuentre al nivel de países desarrollados de menor ingreso. Junto con ello, Chile es el cuarto país más desigual en la región más desigual del planeta $(\text { Gini }=0.51)^{1}$.

Los logros chilenos en materia de superación de la pobreza tienen como trasfondo un modelo de desarrollo económico que se apega estrictamente a los criterios establecidos por el Consenso de Washington, mostrando que este modelo resulta compatible con la mejoría de las condiciones de vida de la población. La relevancia que asume el caso chileno a este respecto viene dada por la capacidad que ha mostrado para mejorar las condiciones de vida sin reducir las agudas desigualdades en la distribución del ingreso. ¿Se acompaña este mejoramiento por una movilidad social o de ingresos que exprese la creación de oportunidades? ¿Qué características se puede decir que asume esta movilidad? En el artículo analizamos las pautas de movilidad ocupacional, las que correlacionamos con los ingresos de la población.

Mirar la sociedad desde el punto de vista de una política social destinada a reducir sustancialmente la pobreza ilumina las áreas de exclusión ("residuos" de la inclusión económica) mientras que pone en la penumbra el proceso de movilidad social ascen-

"La generación de datos originales y su análisis contaron con el apoyo de Conicyt, Proyecto Anillos SOC12.

${ }^{1}$ La afirmación considera solamente los países que cuentan con estadísticas nacionales de ingreso (Therborn 2006). 
dente en el que ha estado involucrada la mayor parte de la población chilena en los últimos veinte años. En la década de 1990, el foco exclusivo en la situación de los pobres oscureció en gran medida el hecho de que el conjunto de la sociedad chilena estaba mejorando sus condiciones de vida. Visto en perspectiva, la reducción de la pobreza forma parte de un amplio proceso de movilidad social ascendente, del cual su superación constituía el caso particular de la movilidad que traspasaba un umbral de ingresos, la "línea de pobreza".

Comprender mejor la evolución de la pobreza y su dinámica demanda moverse desde su análisis focalizado hacia un plano más general, considerando el peso que posee el acceso al mercado de trabajo en el diseño de esta política. La movilidad en el mercado de trabajo viene a constituir el trasfondo de la política social de superación de la pobreza, lo cual se constituyó en la principal razón para estudiar la movilidad ocupacional en Chile (Espinoza 2002). En otras palabras, las características que asume la movilidad en el mercado de trabajo reflejan también las oportunidades que la sociedad ofrece a sus integrantes para alcanzar un nivel adecuado de bienestar (Hout 1988). Al menos una reserva: los pobres que no tienen acceso al mercado de trabajo no fueron considerados en el análisis. No obstante, también hay ocupados que se encuentran bajo la línea de pobreza, lo cual puede indicar que el puro acceso no resuelve el problema de pobreza, pues ésta reflejaría también imperfecciones o segmentación en el mercado de trabajo.

Vincular los problemas de pobreza con los estudios de movilidad ocupacional plantea cambios con respecto a la perspectiva estructural dominante en el análisis de las condiciones sociales en América Latina, que en el lenguaje de la CEPAL se denomina "movilidad estructural" (Solís 2007; Gurrieri \& Sáinz 2003). En efecto, la pregunta por las oportunidades de progreso social abiertas en Chile había sido respondida generalmente en términos de procesos de cambio social en gran escala, especialmente la industrialización y la incorporación de tecnología en los procesos productivos. Los estudios de movilidad ocupacional ponen su foco en los procesos que producen la desigualdad, en particular el cambio en las condiciones de bienestar entre generaciones, así como las barreras a la movilidad en el mercado de trabajo.

Ya en la primera década del siglo XXI se multiplicaron los intentos por vincular los estudios sobre las condiciones sociales de América Latina con la tradición principalmente sajona de los estudios de movilidad (Jorrat 2000; Espinoza 2006; Solís 2007; Torche 2005). En Chile, el intento más sistemático por posicionar los estudios de movilidad en el marco conceptual de la tradición sajona corresponde al trabajo de Florencia Torche. Su estudio tiene como preocupación central discutir la evidencia chilena aparentemente contradictoria, consistente en un alto nivel de desigualdad en la distribución del ingreso acompañada por alta movilidad ocupacional. Tomando como punto de referencia el modelo de fluidez constante formulado por Erikson y Goldthorpe (1992), contrasta los resultados de países industrializados con los resultados obtenidos en Chile el año 2001.

Torche (2005) hizo entrar el caso chileno en el debate internacional sobre la fluidez de las sociedades industriales, cuyos antecedentes se remontan a los años 1950 (Hout 
\& DiPrete 2006). La discusión se refiere al grado de igualdad de oportunidades que poseían los miembros de las sociedades industriales europeas. Chile, en realidad no es, ni probablemente será, un país industrializado de la misma forma que lo eran los países con los cuales se le compara; no obstante, tal comparación puede realizarse para establecer el grado de generalidad de la pauta de movilidad que, en último término, constituye un modelo de la forma que adopta la desigualdad en las sociedades modernas. Desde el punto de vista del debate internacional, la comparación del caso chileno con los países industrializados introduciría alguna claridad respecto a la relación entre la desigualdad de ingresos y la igualdad de oportunidades, un campo sobre el cual no existe evidencia concluyente (Hout \& DiPrete 2006)

De acuerdo con sus resultados, la pauta de fluidez observada en Chile se ajusta al modelo de Erikson y Goldthorpe (1992) si bien para hacer más fuerte su caso, Torche elabora una nueva propuesta a partir de las diferencias significativas observadas en los coeficientes para los casos de Chile y los países que constituyen el "núcleo" en el modelo de movilidad del estudio CASMIN². El modelo propuesto para Chile, resumido en la expresión "desigual pero fluido" caracteriza la movilidad ocupacional en términos de débiles barreras entre sectores de actividad económica, acompañadas por alta movilidad de corto rango.

La caracterización de Torche (2005) diverge de la encontrada en estudios sobre movilidad de ingreso para el mismo período, que entregan la imagen de una sociedad más bien inmóvil, donde existe una tendencia marcada a heredar los niveles de ingreso del padre. Tales estudios han examinado la persistencia en una posición de la distribución de ingreso entre generaciones (herencia), usando como medida la elasticidad de ingreso entre padres e hijos. Los análisis realizados en diversas bases de datos, con diversas metodologías encuentran una elasticidad alta cuyo rango se encuentra entre 0,57 y 0,74 para las edades $25-40$, lo cual resulta alto según estándares internacionales, establecidos por estudios realizados con metodologías comparables (Núñez \& Miranda 2010; 2011), Núñez \& Tartakowsky 2011).

A primera vista los resultados aparecen contradictorios: alta movilidad ocupacional pero baja movilidad de ingresos. Mientras la mirada desde las ocupaciones parece mostrar una sociedad en la cual las oportunidades están abiertas para todos, al mirarla desde el punto de vista del ingreso el panorama aparece casi opuesto. La discrepancia entre movilidad ocupacional e ingresos estáticos se puede asociar con resultados de estudios de movilidad y estratificación social para otros países en los cuales se plantea que el ascenso en términos de posiciones ocupacionales resulta desfasado con respecto a las garantías asociadas con las ocupaciones (Kessler \& Espinoza 2007). Esta y otras formas de desfase o vulnerabilidad formarían parte de un conjunto de procesos y situaciones de "inconsistencia posicional" (Araujo \& Martucelli 2011). En la literatura económica reciente, sin embargo, pueden encontrarse argumentos teóricos que vincu-

${ }^{2}$ El núcleo corresponde a datos de Inglaterra y Francia obtenidos en la década de 1970. 
lan mayor desigualdad de ingresos en corte transversal, con menor movilidad intergeneracional del ingreso (Solon 2002; 2004). Evidencia convergente con este argumento puede encontrarse en Björklund and Jäntti (1997), Corak (2006), Dunn (2007) y Andrews \& Leigh (2008). En la nueva literatura económica sobre movilidad de ingresos resulta teórica y empíricamente menos probable encontrar alta movilidad con alta desigualdad, lo cual establece otro punto de interés para el análisis desarrollado en este artículo.

El artículo busca reconciliar los análisis de movilidad ocupacional y movilidad de ingresos, utilizando para ello evidencia proveniente de la Encuesta Nacional de Estratificación Social (ENES) realizada en 2009. Por cierto, los supuestos de los estudios de movilidad de ingresos son diferentes a los de movilidad ocupacional, por cuanto estiman la movilidad suponiendo que existe un continuo de posiciones, antes que clases separadas por cortes discretos.

La estrategia de análisis establece los cambios en las pautas de movilidad ocupacional desde 2001 a 2009 en términos del modelo de fluidez constante (MFC). Posteriormente, se ajusta un modelo jerárquico a los datos de movilidad, el que permite comparar la evidencia de ingresos con las pautas de movilidad. Si bien las escalas son diferentes, se buscará estimar la pertinencia de la correlación entre la movilidad ocupacional revisada en términos "verticales" y el continuo de ingresos. El análisis permite revisar la congruencia de la segmentación de ingresos en las ocupaciones con los ingresos individuales.

Aun cuando la movilidad de ingresos y la movilidad ocupacional puedan expresarse como desplazamientos "verticales", es decir entre posiciones jerarquizadas, ello no involucra la ausencia de barreras u obstáculos que afecten a la movilidad entre generaciones. El análisis original que se presenta a continuación mostrará por lo tanto la convergencia entre los análisis de movilidad de ingresos y movilidad ocupacional, bajo el supuesto de que un modelo jerárquico permite describir adecuadamente la pauta de movilidad observada en Chile, a la vez que identifica las barreras a esa movilidad entre las generaciones.

\section{Datos del estudio}

La Encuesta Nacional de Estratificación Social (ENES) representa un universo que corresponde a la población mayor de 18 años, residente en el territorio chileno: 12.180.324 de personas al 30 de junio de 2009, según proyecciones del Instituto Nacional de Estadísticas (INE). El diseño muestral utilizado corresponde a uno estratificado por conglomerados en tres etapas: unidad de Muestreo Primaria (UMP) Manzana/Entidad; unidad de Muestreo Secundaria (UMS), Hogar; y unidad de Muestreo Terciaria (UMT), Persona de 18 años y más ${ }^{3}$. El marco muestral utilizado para la selección de las

\footnotetext{
3 "Manzana" y "Entidad" corresponden a un conglomerado de viviendas en las zonas urbanas y rurales, respectivamente; el "Hogar" corresponde al grupo de personas que habitan la misma vivienda y cocinan juntas.
} 
UMP corresponde a la base de datos del Censo de 2002, con actualización de sectores en grandes ciudades. El material cartográfico básico para la identificación de las UMP correspondió a mapas del INE. La selección se realizó mediante un sistema computacional que garantiza aleatoriedad al proceso. En cada UMP sorteada, se empadronaron los hogares existentes y se seleccionaron aleatoriamente cinco hogares. En cada hogar se entrevistó cara a cara a todos los miembros del hogar mayores de 18 años quienes respondieron al mismo cuestionario, salvo el principal proveedor que respondió además un formulario complementario sobre datos generales del hogar. La muestra fue estratificada por región y zona urbana-rural, usando asignación fija. El levantamiento de campo se extendió por 10 semanas, desde el 29 de mayo al 9 de agosto de 2009, a cargo de la empresa Statcom. El tamaño muestral final a nivel de personas fue de 6153 casos, cuyo error muestral máximo alcanza $1,3 \%$ a nivel nacional.

La sección sobre movilidad social de la encuesta ENES contiene información acerca de la ocupación y la escolaridad alcanzada por el padre y la madre del entrevistado/a (o quien desempeñara esa función en caso de no estar presente) cuando éste/a tenía alrededor de 15 años de edad. Los datos obtenidos para hombres y mujeres en el hogar, así como en las preguntas retrospectivas constituyen una innovación con respecto a estudios de movilidad que consideran solamente a los hombres o los jefes de hogar. La disponibilidad de los datos de escolaridad de los padres permite también estimar los niveles de ingreso de éstos (Nuñez \& Miranda 2010; 2011). Para efectos de comparabilidad en el análisis se utilizan los datos de entrevistados cuyas edades se ubican entre los 25 y 64 años, como una forma de centrar el análisis en el período de "madurez laboral", reduciendo el efecto de las fluctuaciones observables en los comienzos y finales de la carrera laboral. El total de casos válidos asciende a 2531, de los cuales 1609 son hombres y 922 mujeres. El nivel de participación femenina en la fuerza de trabajo (37\%) se ubica en el mismo valor que la estimación OCDE, pero aparece algo menor que otros datos (Programa de Naciones Unidas para el Desarrollo 2010), debido a la exclusión de las edades menores de 25 años, en las cuales la proporción de participantes es mayor.

La pieza de información básica en los estudios de movilidad ocupacional es la tabla de origen y destino ocupacional que, para los fines de este análisis, corresponde en el origen a la ocupación del padre, madre o quien fuera el principal proveedor en el hogar del entrevistado/a cuando este/a tenía alrededor de 15 años de edad; y, en el destino, a la ocupación del entrevistado/a al momento de la entrevista. La información sobre los padres se obtiene de forma retrospectiva y constituye un atributo de los entrevistados que no refleja la situación de la fuerza de trabajo en el período de referencia (Duncan 1966). La justificación para enfocarse en la movilidad entre generaciones es que, en este tránsito, se pueden observar los cambios más significativos en la posición social.

La clasificación de ocupaciones utilizada en un estudio de movilidad posee efectos conocidos sobre los resultados del análisis, particularmente en la estimación del volumen de movilidad: clasificaciones más detalladas tienden a registrar mayor movilidad que las clasificaciones con menos categorías. Los estudios de movilidad habitualmente suponen que las categorías corresponden a unas pocas clases sociales de gran tamaño, 
cuyo número en los estudios varía entre 12 y tres grupos ${ }^{4}$. Los datos de ocupación de la encuesta ENES fueron generados por medio de una descripción de las funciones del trabajador en su cargo actual, categoría ocupacional, sector de actividad económica de su empresa, y autoridad dentro del proceso productivo. Las descripciones fueron codificados en el esquema ISCO-88 y posteriormente convertidos a un esquema reducido de categorías ocupacionales.

Existen diversos esquemas para clasificar clases sociales a partir de grupos ocupacionales, aunque la propuesta por Erikson, Goldthorpe y Portocarrero (EGP) se ha convertido en el estándar de facto para comparaciones internacionales al ser adoptada por estudiosos en gran número de países (Erikson \& Goldthorpe 1992; Atria 2004; Ganzeboom \& Treiman 1996). Su característica principal es que no constituye un "continuo" de posiciones sino que supone cortes discretos entre las clases de acuerdo con determinadas características de las relaciones laborales. La clasificación no sigue un esquema teórico particular, aunque puede identificársele como filo-weberiana, al distinguir posiciones de mercado basadas en la propiedad de medios de producción y construir las relaciones contractuales a partir de criterios de autoridad y recursos de mercado.

La categorización EGP distingue en primer lugar el sector económico (agricultura y el resto), posteriormente entre asalariados y propietarios de medios de producción entre los que distingue propietarios corporativos e individuales - y finalmente dentro de los asalariados. Estos últimos son clasificados de acuerdo con su nivel de especialización y la facilidad de control sobre su trabajo, lo cual da origen a la distinción entre asalariados obreros, contratados para desempeñar tareas específicas sobre la base de horas o productos, por contraste con la "clase de servicio", vale decir trabajadores de alta cualificación contratados sin sujeción estricta a productos u horarios; entre ambos extremos se ubican otras clases asalariadas (Goldthorpe \& McKnight 2006). A partir de estos criterios es posible generar entre 11 y tres grupos "anidados" de estatus ocupacional, de los cuales en este artículo se utilizan siete. La operacionalización de las definiciones se realiza a partir de los datos codificados de ocupación a cuatro dígitos, categoría ocupacional y condición de supervisor, ajustando a las peculiaridades de los datos los algoritmos desarrollados por Ganzeboom y Treiman (1996), y considerando las observaciones recientes a este procedimiento (Leiulfsrud et al. 2005) ${ }^{5}$.

\footnotetext{
${ }^{4}$ El análisis de "micro-clases" que incorpora determinantes institucionales especificas no puede abordarse pues requiere muestras mucho mayores que la ENES, (Grusky \& Sorensen 1998; Pakulski 2005).

${ }^{5}$ Los procedimientos de codificación son equivalentes a los que producen los datos presentados por Torche (2005). Comunicación personal de Florencia Torche, 2 noviembre 2010. No obstante, se observan diferencias sensibles en tres categorías: Rutina no-manual (III) que en 2009 supera por 8,1 puntos la estimación de 2001; Pequeños Empresarios, que en 2009 es menor en 4,9 puntos respecto de 2001; finalmente, los operarios calificados aparecen 6,1 puntos por debajo de los valores de 2001. Estas diferencias en la distribución no afectan al tratamiento formal de las pautas de movilidad, como se aprecia al probar los efectos de las estrategias de muestreo en cada encuesta sobre el ajuste y coeficientes de los modelos revisados (ver nota 7).
} 
Tabla 1.

Indicadores de la Fuerza de Trabajo según Grupos de Ocupación. Hombres y mujeres de entre 25 y 64 años de edad.

\begin{tabular}{lccc}
\hline & $\begin{array}{c}\text { Distribución } \\
\text { de los grupos } \\
\text { de ocupación }\end{array}$ & $\begin{array}{c}\text { Mediana } \\
\text { de Escolaridad } \\
\text { (años) }\end{array}$ & $\begin{array}{c}\text { Media } \\
\text { de ingresos } \\
\text { (miles \$ CH) }\end{array}$ \\
\hline I+II: Clase de Servicios & $22,0 \%$ & 15,6 & 202,35 \\
III: Rutina No-Manual & $15,1 \%$ & 12,7 & 123,13 \\
IVab: Pequeño empresario & $17,1 \%$ & 11,3 & 113,05 \\
IVc: Pequeño Agricultor & $4,2 \%$ & 8,0 & 82,94 \\
V+VI: Operario Cualificado & $12,9 \%$ & 12,4 & 98,86 \\
VIla: Operario no Cualificado & $22,6 \%$ & 10,1 & 91,91 \\
VIIb: Trabajador Agrícola & $6,0 \%$ & 8,6 & 83,69 \\
\hline Total & $100,0 \%$ & 12,1 & 124,47 \\
\hline N & 2531 & 2531 & 1138 \\
\hline
\end{tabular}

* Ingresos estimado para hombres de entre 25 y 40 años de edad. Los procedimientos se explican más adelante. Fuente: Encuesta ENES 2009.

Los principales grupos de ocupaciones corresponden a la clase de servicios $(22 \%)$ y los operarios no cualificados $(22,6 \%)$, cuya suma abarca cerca de la mitad de los trabajadores, mostrando un marcado contraste en el mercado de trabajo. La clase de servicios comprende tanto los grupos más favorecidos, grandes empresarios y profesionales exitosos, como la denominada "clase media alta" que incluye otros profesionales de buen pasar. Este grupo concentra profesionales que han realizado estudios superiores y contrasta notablemente con el resto de los trabajadores. El pequeño empresario urbano $(17,1 \%)$ comprende unidades de pequeña escala, incluyendo algunos trabajadores por cuenta propia en emprendimientos estables. El peso de este grupo en la distribución de ocupaciones se ha mantenido relativamente constante a lo largo del tiempo, revelando que se trata de una categoría con fronteras bien delimitadas (Torche \& Wormald 2004). Los grupos de menor peso son los del sector agrícola que alcanzan entre ambos el 10\% de la fuerza de trabajo.

El nivel medio de escolaridad de la fuerza de trabajo alcanza el equivalente a la educación media completa y resulta consistente con datos de otras fuentes (Ministerio de Educación de Chile 2009). Los niveles más altos de escolaridad, cercanos a los 16 años, se encuentran en la clase de servicios indicando el acceso a educación universitaria de sus integrantes. Los niveles más bajos, equivalentes a educación básica, se encuentran en el sector agrícola, tanto en pequeños agricultores como entre trabajadores agrícolas. En los restantes grupos ocupacionales los valores se encuentran cercanos al nivel medio. 
Los ingresos medios de los grupos ocupacionales muestran su nivel mayor en la clase de servicios, casi dos tercios por sobre la media de los ingresos. Los trabajadores no manuales de menor cualificación así como los pequeños empresarios urbanos obtienen ingresos cercanos a la media. Los trabajadores manuales, con independencia de su cualificación obtienen ingresos claramente por debajo de la media, superando en forma clara, sin embargo, los ingresos de los pequeños agricultores y los campesinos sin tierra, grupos en los que se observan los menores niveles de ingresos.

\section{NiVELES Y PAUTAS DE MOVILIDAD OCUPACIONAL}

El "modelo de fluidez constante" propuesto por Erikson y Goldthorpe (1992) establece una pauta de movilidad que se hipotetiza común a las sociedades industrializadas. El modelo identifica efectos de jerarquía, herencia, sector y afinidad, que operan en los procesos de movilidad, los cuales especifica con ocho parámetros.

El efecto de jerarquía divide la tabla en tres estratos que reflejan las clases más deseables (Servicios, I+II), las menos deseables (Campesinos, VIlb y Manuales baja cualificación, Vlla); el tercer estrato de las clases intermedias corresponde a todos los grupos ocupacionales restantes. El efecto de jerarquía se especifica con dos parámetros que identifican barreras entre estratos: movimientos de un paso o corta distancia y movilidad de dos pasos o larga distancia. Tanto ascenso como descenso están modelados por el mismo parámetro, que se hipotetiza debe ser negativo, es decir, las probabilidades de encontrar movilidad entre estas barreras es menor que en una situación de neutralidad (cuando el valor esperado bajo el modelo, es igual al valor observado en la medición). El efecto de herencia recoge la propensión de los individuos a mantenerse en la posición de origen y comprende tres parámetros: uno para la propensión general a heredar la posición del padre, otro para la herencia del autoempleo y un tercero para las posiciones de pequeño propietario agrícola. El efecto de sector se refiere a la propensión a trasladarse entre posiciones agrícolas y no agrícolas y se hipotetiza que debiera ser negativo. Finalmente, el modelo considera dos efectos de afinidad, vale decir, las probabilidades que dos grupos de status ocupacional muestren discontinuidad o desarrollen vinculaciones sociales de diverso tipo. El primer efecto de afinidad, llamado de afinidad negativa, especifica la improbable vinculación de origen y destino entre las posiciones ocupacionales extremas; el segundo efecto reúne diversas vinculaciones 0 afinidad positiva, que no siguen una pauta general, pero que poseen una base empírica: entre trabajadores no manuales $(\mathrm{I}+\mathrm{II}, \mathrm{III})$; dentro de los trabajadores manuales (V, VI y VIla); entre quienes poseen capital (IVab y IVc; I y IVab); finalmente, los trabajadores manuales no cualificados (VIla) constituirían el destino más frecuente para quienes provienen de ocupaciones agrícolas (IVc y VIllb). 


\section{AJUSTE DEL MODELO DE FLUIDEZ CONSTANTE}

El modelo de fluidez constante se especifica como un modelo log-lineal topológico cuyos niveles están parcialmente traslapados, de forma que incluye ocho parámetros para dar cuenta de los efectos indicados en la descripción cualitativa anterior ${ }^{6}$. Bajo este modelo, la frecuencia esperada para cada posición de destino condicional al origen puede presentarse siguiendo la notación corriente para modelos log-lineales:

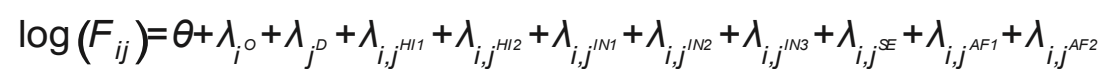

Donde $\log \left(F_{i j}\right)$ es el logaritmo natural de la frecuencia esperada, $\theta$ es un factor de escala, $\lambda_{\mathrm{i}} \mathrm{y} \lambda_{\mathrm{j}}$ representan los efectos de origen y destino, respectivamente. Los $\lambda_{\mathrm{i}, \mathrm{j}}$ corresponden a los efectos de los parámetros del modelo según la indicación del superíndice. La ecuación que especifica un modelo log-lineal corresponde a una regresión lineal múltiple con vínculo Poisson, cuya variable dependiente son las frecuencias de cada casillero de la tabla (Fox 2008). Los cálculos se realizaron utilizando la aplicación "glm" del paquete estadístico R (R Development Core Team 2010).

La razón de verosimilitud (L2) es un estadístico de ajuste que evalúa la calidad de los modelos ubicados entre la situación de independencia (que rara vez ajusta los datos) y el modelo saturado (el cual ajusta por necesidad matemática, utilizando todos los grados de libertad disponibles). Los modelos ajustan cuando utilizan menos grados de libertad que el modelo saturado, sin mostrar diferencias significativas con éste. Como habitualmente existe más de un modelo que ajusta los datos, la selección recae en el investigador. En muestras de gran tamaño es raro encontrar modelos que ajusten según el criterio probabilístico convencional, por lo que se recomienda utilizar como alternativa el Criterio Bayesiano de Información o BIC (Treiman 2009). En los últimos años el índice de disimilitud ha adquirido mayor difusión, incluso como alternativa al BIC (Kuha \& Firth 2011). La tabla 2 presenta la estadística de ajuste general del modelo de fluidez constante (MFC) y la evaluación de la bondad de ajuste, utilizando los datos de la encuesta ENES según el modelo original y luego con las especificaciones propuestas por Torche (2005) para Chile.

\footnotetext{
${ }^{6}$ Para facilitar la comparación con publicaciones que utilizan este modelo se nombran los parámetros según las abreviaturas usadas en inglés: HI: Jerarquía, IN: Herencia, SE: Sector, AF: Afinidad.
} 
Tabla 2.

Estadísticas de ajuste del modelo de fluidez constante (MFC) para datos ENES. Chile 2009: Fuerza de trabajo ambos sexos 25-64 años de edad.

\begin{tabular}{lccccc}
\hline modelos & L2 & gl & BIC & $\begin{array}{c}\text { L2/L2 } \\
\text { independencia }\end{array}$ & $\begin{array}{c}\text { Índice } \\
\text { disimilitud (\%) }\end{array}$ \\
\hline Independencia & 768,04 & 36 & 486 & 1 & 19,2 \\
MFC (Original CASMIN) & 87,633 & 28 & -132 &, 11 & 6,9 \\
MFC (Especificación Torche A) & 89,945 & 28 & -129 &, 12 & 6,9 \\
MFC (Especificación Torche B) & 84,914 & 27 & -127 &, 11 & 6,3 \\
\hline
\end{tabular}

Fuente: Elaboración propia.

Los datos se encuentran lejos de una situación de independencia, por lo que puede probarse el ajuste de modelos alternativos. El modelo de fluidez constante en su versión original produce un buen ajuste de los datos de ENES tomando en cuenta el valor negativo del BIC, la reducción del valor de L2 en 89\%.Las especificaciones propuestas por Torche (2005) para Chile no mejoran significativamente el ajuste ${ }^{7}$, e incluso el valor del BIC indica que en estos datos su modelo resulta menos probable que el modelo de fluidez constante en su versión original. Si bien la especificación B mejora levemente el índice de disimilitud, los restantes criterios no aconsejan abandonar el modelo original.

Identificar la pauta de movilidad requiere considerar el valor de los parámetros del modelo por separado, los cuales se presentan comparando su comportamiento en tres contextos diferentes: datos para Chile de la encuesta ENES, medición de 2009; datos para Chile de Torche (2005), medición de 2001, y datos del núcleo presentados por Erikson y Goldthorpe (1992) ${ }^{8}$.

${ }^{7}$ Los procedimientos de ajuste para el MFC fueron realizados en los datos del 2009 bajo las restricciones que impone el diseño muestral de 2001 (solamente hombres jefes de hogar, excluyendo regiones extremas). Los coeficientes obtenidos usando estas restricciones son los siguientes: $\mathrm{HI} 1=-0,27 ; \mathrm{HI} 2=-0,4 ; \mathrm{IN}=0,39$; IN2=0,54; IN3=-0,13; SE=-1,06; AF1=-0,2; AF2=0,34; los cuales no muestran diferencias significativas con los coeficientes calculados para la totalidad de la muestra. Adicionalmente, se ensayó también excluir el sector agrícola, manteniéndose la estabilidad en los resultados.

${ }^{8}$ Uno de los revisores del artículo anotó que los valores del BIC reportados por Torche (2005) para el Core-model original no corresponden a la fórmula convencional de cálculo; en realidad, la diferencia proviene de que en la fórmula convencional ella reemplazó el total de casos por el número de casilleros en la tabla. Al utilizar el total de casos, el modelo de fluidez constante ajusta los datos del 2001. Puede entenderse que prefirió redefinir los parámetros para describir la pauta de movilidad chilena con más precisión, a costa de la parsimonia del modelo. No obstante, el refinamiento al modelo de fluidez constante propuesto por ella tiene como punto de partida una apreciación errada del ajuste estadístico. 
Tabla 3.

Comparación de parámetros del modelo de fluidez constante en tres contextos.

\begin{tabular}{lcccccccc}
\hline Modelos & HI1 & HI2 & IN1 & IN2 & IN3 & SE & AF1 & AF2 \\
\hline MFC Chile 2009 (ENES) & $-0,30$ & $-0,63$ & 0,23 & 0,36 & 0,11 & $-1,16$ & $-0,71$ & 0,23 \\
MFC Chile 2001 (Torche) & $-0,12^{* * *}$ & $-0,65$ & $0,41^{* *}$ & 0,39 & 0,68 & $-0,38^{* * *}$ & $-0,76$ & $0,33^{* *}$ \\
MFC Core Model & $-0,22^{*}$ & $-0,45^{* *}$ & $0,43^{* * *}$ & $0,84^{* * *}$ & $1,01^{* *}$ & $-1,06$ & $-0,83$ & $0,45^{* * *}$ \\
significación con respecto ENES & ${ }^{* * *} \mathrm{P}<, 001$ & & ${ }^{* *} \mathrm{P}<, 01$ & & ${ }^{*} \mathrm{P}<, 1$ & & & \\
\hline
\end{tabular}

Fuente: Elaboración propia con datos ENES.

Los tres modelos presentan coeficientes significativos cuyos efectos van en la misma dirección, pero con diferencias de magnitud que llegan a ser significativas. En los datos ENES para Chile en el año 2009, el coeficiente para Sector $(-1,16)$ resulta ser el mayor en términos absolutos, lo cual indica oportunidades más bajas de movilidad entre la agricultura y los sectores industrial y de servicios en la economía. Le sigue el efecto de Afinidad negativa entre categorías extremas (AF1 = -,71) y finalmente la movilidad de dos pasos o larga distancia en la Jerarquía (HI2=-,63). Los tres coeficientes de mayor tamaño son negativos y están referidos, bajo diversas formulaciones, a la escasa opción de movilidad entre los extremos de la distribución de categorías ocupacionales; vale decir entre la clase de servicios y los asalariados agrícolas, la cual tiende a solaparse con la separación sectorial. En la suma de los coeficientes para cada uno de los efectos, la diferencia sectorial continúa en primer lugar, seguido por los efectos de jerarquía $(-, 93)$. Los efectos de herencia $(, 70)$ indican una propensión menor a la obtenida por simple oportunidad a mantener la posición ocupacional de los padres.

Al comparar los resultados con los datos disponibles para Chile en el año 2001 se aprecian puntos de convergencia y separación. En común se encuentran: menor propensión a la herencia ocupacional entre autoempleados y propietarios agrícolas, baja propensión a la movilidad de dos pasos (o de larga distancia) y el peso de la afinidad negativa. Las diferencias alcanzan dos coeficientes clave en la argumentación de fluidez en la movilidad para Chile: movilidad de un paso o corta distancia en la jerarquía (HI1) y las barreras sectoriales (SE). En lo que se refiere a movilidad de corta distancia en los datos para Chile del $2001(-, 12)$, el paso desde posiciones intermedias hacia las superiores, sin estar abierto, aparece más probable que en $2009(-, 30)$, o que en los datos europeos $(-, 22)$. Igualmente el bloqueo de acceso a posiciones intermedias desde las más bajas poseía una barrera menor en el 2001. En lo referido a las barreras entre sectores de actividad económica, el valor encontrado en 2001 
$(-, 38)$ resulta ser significativamente más bajo que en $2009(-1,06)$ y que en el contexto europeo $(-1,16)$. La movilidad chilena en 2009 aparece también más trabada porque tampoco se aprecia propensión a la movilidad de larga distancia, de forma que los efectos de jerarquía adquieren un relevante segundo lugar, tras los de sector.

En 2001, el principal efecto correspondía a la herencia ocupacional, al igual que en el contexto europeo, si bien las oportunidades de heredar la posición ocupacional eran más bajas en Chile $(1,48)$ que en Europa $(2,28)$. Los efectos de herencia son menores aún en los datos del $2009(, 70)$ debido especialmente a los bajos niveles observados en las ocupaciones agrícolas. Lo anterior tiene su origen en el escaso peso que posee la afinidad positiva (AF2) en los datos del 2009, la cual no logra compensar los efectos negativos que introduce la distancia sectorial ${ }^{9}$. De hecho, la presencia de barreras sectoriales hace que la movilidad sea menos de un tercio de lo que correspondería en una situación de movilidad neutral $\left(e^{-1,16}=0,31\right)$. Ello afecta particularmente a la movilidad desde y hacia posiciones en el sector agrícola.

\section{ASPECTOS JERÁRQUICOS DE LA MOVILIDAD OCUPACIONAL}

La pauta de movilidad chilena en el 2009 se acerca a la europea en términos de la relevancia de la barrera entre sectores de actividad económica y el peso que poseen los factores jerárquicos; la principal diferencia se encuentra en el escaso peso que posee la herencia ocupacional en Chile. De estos rasgos, el Chile del 2001, comparado con Europa, mostraba menor peso de la herencia ocupacional, así como mayor peso del elemento jerárquico, descontando cierta mayor propensión a la movilidad entre posiciones intermedias y las contiguas. La principal diferencia de los datos del 2001, tanto con los datos del 2009 como con los europeos, reside en el escaso peso de las barreras sectoriales.

Existen diversas razones que pudieran explicar la diferencia en el comportamiento de los datos entre 2001 y 2009, tanto relativos a la confiabilidad de los mismos (diseño de la muestra, criterios de codificación), presencia de mujeres en el análisis, o bien otros relativos al contexto social y económico en el cual se aplicó la medición. Aquellos referidos a los datos fueron descartados por medio de diversas pruebas que no viene al caso exponer con detalle. Lo que sigue explora la dimensión jerárquica como una forma de representar parsimoniosamente una pauta común presente en ambas mediciones. Tanto los datos del 2001 como los de la encuesta ENES para el 2009 pueden presentarse con un modelo que considera solamente los efectos jerárquicos, que modelan adecuadamente los datos.

\footnotetext{
${ }^{9}$ Es posible que algunas afinidades no se apliquen para Chile.
} 
Erikson y Goldthorpe (1992:139) argumentaron explícitamente, sobre bases empíricas y teóricas, contra la conveniencia de enfocar el análisis sobre su componente jerárquico. Empíricamente, el ajuste de los datos basado en un enfoque jerárquico resultaba más deficiente que el MFC porque los efectos que intentaba modelar no eran de tipo lineal. Otro aspecto relevante es que si el ranking de categorías ocupacionales no coincidía entre países, las comparaciones basadas en un principio jerárquico perderían relevancia sustantiva (Hout \& DiPrete 2006). De todas formas, Erikson y Goldthorpe (1992) dejaron abierta la posibilidad de modelar jerárquicamente cuando la preocupación sustantiva de un estudio así lo requiriera.

Desde un punto de vista conceptual, los efectos de sector y herencia, no debieran ser considerados como parte de una jerarquía. En particular, ubicar a los propietarios al mismo nivel de los trabajadores en el sector agrícola contravendría su jerarquización en términos de status. Un segundo aspecto en esta misma línea, se refiere al carácter heterogéneo o multidimensional que poseerían las jerarquías presentes en los datos CASMIN, como lo revelan las zonas de movilidad de corto alcance para obreros y empleados. El aspecto empírico del problema se puede verificar contrastando la homogeneidad en el orden en las categorías ocupacionales. Los datos chilenos permiten hacerlo en 2001 y 2009, para luego efectuar la comparación con las estimaciones de ingresos. Los análisis se realizan solamente para Chile, por lo cual no hay pretensión de generalizar a otros países la relevancia que se le asigna a la dimensión jerárquica en este trabajo

La reservas respecto a la estratificación del sector agrícola deben revisarse de acuerdo con su contexto histórico, lo cual puede afectar a las decisiones de ubicación en una escala de categorías ocupacionales. La pequeña producción agrícola de los farmers europeos se refiere a unidades equivalentes a las pequeñas empresas urbanas. Se trata de propietarios de tierra y medios de producción que trabajan con su familia $y$, eventualmente, con asalariados produciendo bienes comercializables de forma competitiva. Estas unidades se diferencian de la explotación agrícola a gran escala, que sigue las pautas de una empresa de tipo capitalista. En los datos chilenos del 2009, el pequeño propietario agrícola posee recursos de producción como tierra, maquinaria o animales, pero rara vez contrata mano de obra, dependiendo principalmente de su propio trabajo y de el de sus familiares.

\section{AJUSTE DEL MODELO JERÁRQUICO}

Los modelos RC (fila-columna) ofrecen una síntesis adecuada de los datos de una tabla de movilidad dominada por la verticalidad. El modelo RC es de tipo log-multiplicativo y permite utilizar una escala que representa la distancia entre las categorías, sea ésta propuesta por el investigador, o bien, otra obtenida como variable subyacente a partir 
de la configuración de los datos; en este caso, la pauta de movilidad presente en la tabla de origen-destino ocupacional (Goodman 1985). La Fórmula 2 contiene un término multiplicativo que especifica la interacción entre las escalas para origen y destino en la tabla:

$$
\log \left(F_{i j}\right)=\theta+\lambda_{i} \circ+\lambda_{j}+\varphi \psi
$$

En la fórmula $F_{i j}$ corresponde a la frecuencia esperada bajo el modelo, $\lambda$ representa los efectos de origen o destino según el superíndice, $\varphi$ y $\psi$ los puntajes de escala para origen y destino. El modelo permite calcular un coeficiente que sintetiza las chances de movilidad entre una categoría y otra que, bajo los criterios del orden obtenido, resulta ser la misma para toda la tabla. En los modelos RC homogéneos, $\varphi$ y es igual a $\psi$ lo cual involucra usar la misma escala para origen y destino. Los datos de ajuste con los datos de 2009 y 2001, se presentan en el Cuadro 4. Los cálculos se realizaron utilizando la aplicación gnm del paquete estadístico R (Turner \& Firth 2010).

Cuadro 4.

Estadísticas de ajuste del modelo RCII para movilidad ocupacional chilena 2009.

\begin{tabular}{lccc}
\hline Modelo & L2 & gl & BIC \\
\hline RCII Heterogéneo 2009 & 48,08 & 18 & $-92,97$ \\
RCII Homogéneo 2009 & 68,78 & 23 & $-111,46$ \\
RCII Heterogéneo 2001 & 37,42 & 18 & $-107,71$ \\
RCII Homogéneo 2001 & 46,43 & 23 & $-137,73$ \\
\hline
\end{tabular}

Fuente: Elaboración propia con datos ENES.

Este tipo de modelo puede considerarse una aproximación por máxima verosimilitud al escalamiento óptimo, pues identifica una variable latente en la pauta de asociación de los datos (Goodman 1985; Agresti 2002). La variable latente corresponde a las distancias relativas entre las diversas categorías, dada una determinada pauta de asociación, permitiendo de esta forma identificar trayectorias de movilidad comunes o discrepantes, según los puntajes se encuentren cercanos o lejanos. La existencia de puntajes no involucra per se continuidad en las categorías, sino solamente un posicionamiento relativo —orden y distancia - de unas con respecto a las otras.

El Gráfico 1 muestra los puntajes asociados con las escalas bajo supuesto de homogeneidad en 2001 y 2009, mapeados en un espacio común. Las comparaciones de posición entre ambas muestras puede visualizarse como una proyección de los puntos 
Gráfico 1.

Dimensión vertical de la movilidad ocupacional en Chile

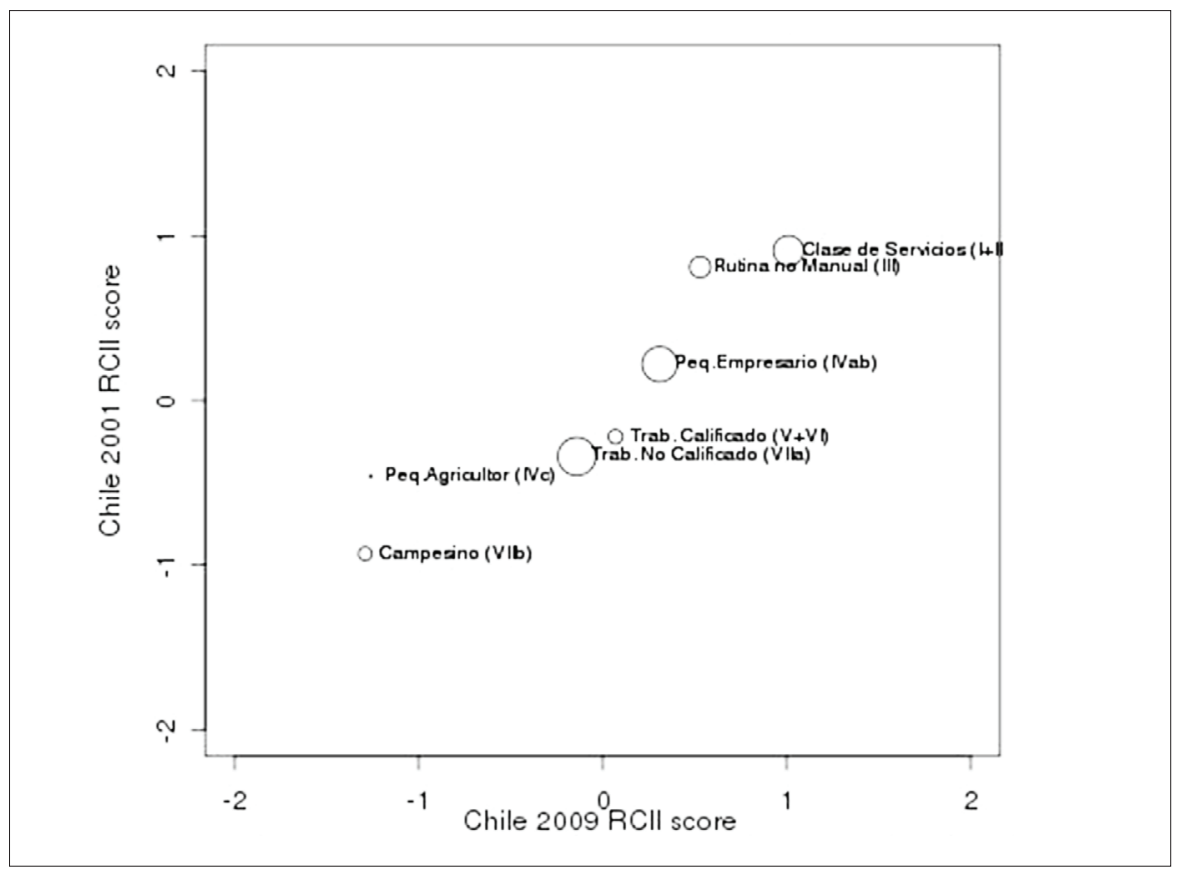

Fuentes: Encuesta Enes 2009. Elaboración propia datos de Torche (2005).

sobre el eje correspondiente. El tamaño de los puntos refleja el peso de la categoría en 2009, como porcentajes de la fuerza de trabajo.

La gráfica de los puntajes en cada muestra permite apreciar la alta asociación entre ambas distribuciones, expresada en el idéntico orden de las categorías, variando solamente las distancias entre clases. Tanto a comienzos como a final de la década, la clase de servicios (I+II) ocupa con claridad uno de los extremos de la distribución, mientras que el trabajador agrícola (Vllb) se ubica indudablemente en el otro extremo. El agricultor propietario (IVc) aparece más cerca del campesino en los datos del 2009, mientras que el 2001 se ubicaba más cerca de los trabajadores. En ambas muestras su posición le aleja de los pequeños empresarios urbanos y lo acerca a los trabajadores de menor status. En las posiciones intermedias, el orden es exactamente igual en ambas muestras.

En cuanto a la separación entre niveles de jerarquía pueden apreciarse discrepancias entre ambas mediciones. En 2001, la clase de servicios (I+II) aparece más cercana a los trabajadores en tareas rutinarias de gestión (III), mientras que al final de la década estas posiciones aparecen más distanciadas. Ello refleja la presencia y ausencia de movilidad 
ascendente de corto rango al comienzo y al final de la década, respectivamente. En el otro extremo, la posición del pequeño agricultor y el asalariado agrícola son prácticamente indistinguibles al final de la década, lo cual marca un contraste con el comienzo, cuando los pequeños agricultores aparecían más cerca de los trabajadores manuales $(\mathrm{V}+\mathrm{VI}$ y VIla). En suma, comparada con el año 2001, en la muestra de 2009 se observan distancias mayores de las categorías extremas con sus posiciones contiguas.

El principal cambio que se observa hacia el final de la década consiste en la cercanía de las posiciones de campesino y pequeños agricultores. El corte sectorial aparece muy claro, cercano a los niveles europeos y mucho más marcado que en los datos del 2001. La disponibilidad de tierra, maquinarias 0 animales no garantiza a la familia del pequeño agricultor oportunidades demasiado diferentes a las que experimentan los trabajadores del campo, con los cuales comparten un destino de inestabilidad laboral y difíciles condiciones de vida. De forma similar, en el otro extremo, la ubicación del límite inferior de la clase media entra también en debate, pues de acuerdo con los datos del 2001, los trabajadores en tareas rutinarias de gestión podían considerarse en conjunto con la clase media alta, lo cual no ocurre en el 2009.

El corte sectorial entre la agricultura y el resto de la producción nacional requiere ponerlo en el contexto histórico de la modernización capitalista que experimentó la agricultura desde mediados de los años 1970 (Kay \& Silva 1992). En efecto, la expropiación de tierras realizada bajo la reforma agraria entre 1965 y 1973 dio como resultado un volumen significativo de tierras bajo control estatal. La política de la dictadura consistió en entregar parte de la tierra a campesinos en parcelas de propiedad individual; rematar otra parte de las tierras, las que fueron adquiridas por empresarios que aplicaron formas de producción capitalista, particularmente el régimen de trabajo asalariado e innovación tecnológica para cultivo intensivo de productos destinados a la exportación. Aunque parte de las tierras también volvió a manos de los latifundistas que las poseían antes de la reforma agraria, el orden agrario resultante no guardaba relación con el tradicional. El sector de pequeños agricultores que sobrevive a este proceso lo hace normalmente en suelos de baja calidad, sin alcanzar competitividad en algún tipo de producción.

El orden agrario se consolidó en los años 1990 dada la competitividad alcanzada por las empresas agrícolas exportadoras, de forma que sus unidades productivas y la fuerza de trabajo ocupada en ellas muestran estabilidad hasta el presente. Si bien Torche (2005) afirma que tal mercantilización del mundo agrario contribuye a reducir las barreras sectoriales, no presenta el proceso o mecanismo a través de lo cual ello se produciría. En realidad, los bordes del sector agrario se demarcan con nitidez frente a las actividades económicas que caracterizan a la producción industrial, minera 0 de servicios. No debe confundirse esto con la fluidez con la cual la población agraria se mueve entre asentamientos urbanos y explotaciones agrícolas. En efecto, el fin de la pequeña agricultura liberó suelos de alta rentabilidad para 
la agricultura de exportación, pero no expulsó a la fuerza de trabajo que dependía de ellos mucho más lejos que ciudades pequeñas e intermedias próximas a las nuevas explotaciones. De esta forma, una fuerza de trabajo agraria localizada en asentamientos urbanos de tamaño reducido es una marca distintiva del nuevo orden rural, por lo cual un mayor grado de urbanización no va necesariamente en desmedro de la producción agrícola.

\section{¿SINTETIZA EL INGRESO MONETARIO LA DIMENSIÓN VERTICAL DE LA MOVILIDAD OCUPACIONAL?}

Los datos de ambas muestras permiten ubicar la pauta de movilidad ocupacional sobre una dimensión continua. De acuerdo con esto, existiría escasa diferenciación "horizontal" en los niveles de ingreso, siendo la movilidad ocupacional principalmente una cuestión de ascenso o descenso sobre una misma escala. El problema sustantivo reside en asociar esta dimensión con otras expresiones de jerarquía. Un candidato obvio para ello es el ingreso monetario de los trabajadores, aunque existen otros: la escolaridad o el status socioeconómico. Dada la relevancia que posee el ingreso del trabajo en la caracterización de la desigualdad, consideraremos este aspecto con mayor detalle. En primer lugar estableciendo la movilidad de ingresos y posteriormente comparando las dimensiones verticales de las categorías ocupacionales.

Los estudios de movilidad de ingresos deben estimar los ingresos no observados de los padres a partir de información retrospectiva sobre sus características socioeconómicas, reportada por los entrevistados. En el caso de los entrevistados también resulta pertinente utilizar este tipo de estimación, porque ofrece una mejor aproximación a los ingresos permanentes del trabajador (Nuñez \& Miranda 2010; 2011). El uso de ingresos permanentes o de largo plazo permite reducir el sesgo al estimar la elasticidad de ingresos de padres a hijos sobre la base de los ingresos corrientes (Solon 1992; Zimmermann 1992).

Los ingresos fueron estimados con el método conocido como two-sample two-stage least squares (TSTSLS). El procedimiento utiliza dos muestras diferentes: la de una generación más antigua (padres) para estimar la ecuación de salarios, obteniendo coeficientes asociados con los determinantes del ingreso. En este caso utilizamos solamente la escolaridad reportada. Los coeficientes obtenidos se aplican sobre la información retrospectiva de los padres en la muestra de los entrevistados (hijos), para obtener una predicción de los ingresos de sus padres. Formalmente, el ingreso corriente de los padres para un período $t$ viene dado por:

$$
Y_{i t}^{p}=Y_{i}^{p}+\alpha_{1} E d a d_{i t}^{p}+\alpha_{2}\left(\operatorname{Edad}_{i t}^{p}\right)^{2}+\mu_{i t}^{p}
$$


El último término incorpora las fluctuaciones transitorias del ingreso corriente y los errores de medición. La edad controla los efectos del ciclo de vida sobre los ingresos. El ingreso permanente de los padres puede describirse con un vector $Z$ de características socio-económicas; de esta forma:

$$
Y_{i t}^{p}=Z_{i}^{p}+v_{i}^{p}
$$

Donde el último elemento es un término no observado, independiente de $Z$, que afecta el ingreso permanente. Al combinar las ecuaciones 3 y 4 obtenemos la predicción del ingreso permanente de los padres.

$$
Y_{i}^{p}=Z_{i}^{p} \gamma^{+} \alpha_{1} \operatorname{Edad}_{i}^{p}+\alpha_{2}\left(\operatorname{Edad}_{i}^{p}\right)^{2}+\mu_{i}^{p}+v_{i}^{p}
$$

En nuestro estudio el vector $Z$ incorpora la escolaridad de los padres y su categoría ocupacional. Los niveles de escolaridad se introdujeron en forma de variables mudas: educación primaria completa, educación secundaria completa, educación técnica completa y educación universitaria completa. La categoría de referencia corresponde a educación primaria incompleta o sin educación formal. Las categorías de ocupación corresponden a: empleadores, trabajadores por cuenta propia, asalariados y servicio doméstico. A partir de la estimación anterior es posible obtener la elasticidad intergeneracional de ingresos utilizando la predicción del ingreso del padre como regresor.

La elasticidad obtenida con los datos de la encuesta ENES alcanza ,55, cifra cercana a la obtenida con los datos de la encuesta CASMIN-Chile 2001, y que resulta elevada en relación a la evidencia internacional. Ello indica una alta propensión a la herencia de los niveles de ingresos de los padres en sus hijos. La excepcionalidad destacada por Torche (2005) para el caso chileno —alta movilidad ocupacional junto con alta desigualdad de ingresos- debiera moderarse a partir de estos análisis. Aparentemente, la movilidad ocupacional no va acompañada por cambios significativos en los niveles de ingresos de las personas, de forma que la jerarquía de ingresos resulta estable en el tiempo.

El segundo aspecto a considerar corresponde a verificar la asociación existente entre la escala jerarquizada de movilidad ocupacional y los niveles de ingresos para estas mismas categorías. Para ello se reemplazan los puntajes óptimos del modelo RCll con el ingreso estimado en cada grupo ocupacional; si el modelo ajusta puede decirse que una escala es equivalente a la otra. El reemplazo contempló diversas transformaciones del dato de ingresos: logaritmo, media, mediana y porcentajes de casos sobre distintos umbrales de ingresos. De entre ellas, el ingreso medio de los grupos ocupacionales ofrecía el mejor ajuste. En ninguno de los casos, sin embargo, fue posible obtener un ajuste satisfactorio. Tampoco fue posible ajustar los datos de movilidad utilizando escalas de escolaridad, prestigio de las ocupaciones y status socio-económico de los hogares. En suma, si bien 
Gráfico 2.

Dispersión entre movilidad ocupacional e ingresos.

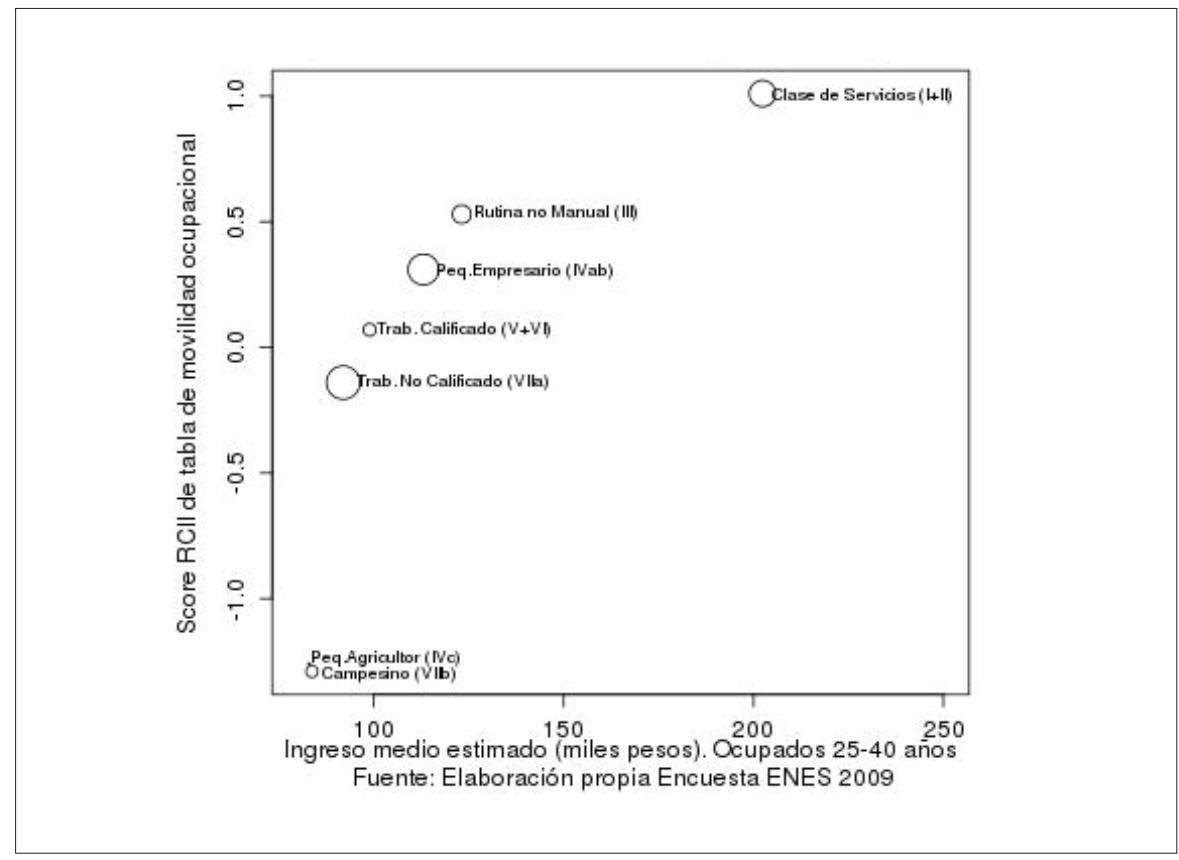

Fuente: Elaboración propia. Encuesta ENES 2009.

la movilidad ocupacional puede representarse en términos "verticales", vale decir como "subidas y bajadas", tal dimensión comprende aspectos diferentes al ingreso de las personas.

Las razones por las cuales no se logra un ajuste del modelo pueden apreciarse a través del examen visual de sus propiedades de orden como de la forma de la asociación. El Gráfico 2 muestra la dispersión entre la escala de puntajes para la movilidad ocupacional obtenidos con el modelo RCll y los ingresos medios estimados para las categorías de ocupación en la muestra de 2009. El tamaño de los puntos es proporcional al peso de los grupos ocupacionales en la fuerza de trabajo.

La gráfica muestra una asociación no lineal entre ambas medidas, las cuales poseen exactamente el mismo orden. La movilidad ocupacional y el ingreso jerarquizan las ocupaciones de la misma forma, pero sin alcanzar una relación lineal. Desde el punto de vista de la desigualdad de ingresos, la mayor discrepancia viene dada por la distancia entre la Clase de servicios y las restantes. Tal distancia también queda reforzada por la diferencia en las pautas de movilidad con respecto a otras ocupaciones. Desde el punto 
de vista de la movilidad ocupacional, la mayor distancia se observa entre las categorías agrícolas y el resto, aunque sin diferencias notorias en los niveles de ingreso. Los grupos que ocupan las posiciones extremas son grupos de alta herencia ocupacional intergeneracional, por lo que la distancia también puede asociarse con barreras. Mientras que en la clase de servicio la barrera puede interpretarse como jerarquías derivadas de recompensas asociadas con la posición, en el otro extremo se mezclan los elementos sectoriales con los de jerarquía.

\section{CONCLUSIONES}

El panorama de la movilidad social en Chile durante la primera década del siglo XXI muestra un paso desde mayor a menor fluidez. El aspecto más llamativo reside en la desaparición de las zonas de movilidad de corto rango, dando origen a una pauta en la cual la herencia constituye la única situación en la cual los valores observados están por encima de los esperados bajo el modelo. En el resto de la tabla, los coeficientes indican que se debe esperar menos movilidad que la prevista por el modelo. Descontando posibles discrepancias en los dispositivos de medición entre ambos estudios, existe una pauta común, expresada en un componente jerárquico, cuya modelación entrega un mismo orden en los dos momentos. Las distancias entre categorías no son las mismas, sin embargo. A comienzos de la década, las categorías ocupacionales extremas se encontraban más próximas a sus categorías contiguas que al final de la década, revelando un tránsito hacia cierta clausura en los destinos ocupacionales más y menos favorecidos de la distribución.

La menor distancia entre las posiciones extremas y sus contiguas que presentan los datos del 2001 no reduce la fuerza de esta caracterización, particularmente en la relación entre los extremos. En efecto, la movilidad de larga distancia, esto es ascenso y descenso entre las clases altas y bajas, aparece igualmente alto en ambas mediciones para Chile y significativamente más bajo que en la medición europea. De aquí deriva la posibilidad de ajustar exitosamente los datos chilenos con un modelo jerárquico, en el cual el corte sectorial se confunde con los destinos menos favorecidos.

Las diferencias jerárquicas coinciden con los extremos de la distribución de los ingresos, aunque no se reflejan exactamente en una relación lineal. Desde el punto de vista del ingreso existirían solamente dos grupos: la clase de servicio y el resto. La distancia entre categorías establecida por la movilidad ocupacional muestra la presencia de barreras "abajo" y "arriba", que obstaculizan la salida desde las posiciones más desfavorecidas, así como el acceso a las posiciones más altas. Se establecen así tres grupos que pueden concebirse como destinos comunes en los procesos de movilidad, vale decir como clases sociales en un sentido weberiano. La pauta de movilidad permitiría distinguir, además, dos estratos dentro de los grupos intermedios: quienes se encuentran saliendo desde posiciones más desaventajadas y quienes compiten por el acceso a 
posiciones en la clase de servicio. La clave del acceso, especialmente a las posiciones asalariadas, reside en la cualificación escolar universitaria (Espinoza \& Barozet 2009).

El significado de los grupos ocupacionales intermedios adquiere otra dimensión a la luz de estas consideraciones. Los grupos ubicados entre los extremos de una ordenación jerárquica de categorías ocupacionales se encuentran entre cortes discretos en tal estratificación, los cuales actúan como barreras, tanto a la salida desde las posiciones más bajas como en el acceso a las posiciones más altas. La concepción de la clase media como un enlace entre sectores altos y bajos en un camino de ascenso social resulta cuestionada en este tipo de configuración. Incluso, el sector menos cualificado de la clase media ya no se encuentra, como en la clase media del siglo XX, a la expectativa del ascenso, sino que se enfrenta de forma permanente al riesgo de descenso.

A pesar de la reproducción en las posiciones ocupacionales, la movilidad ocupacional continúa siendo comparativamente alta en Chile, la cual expresaría pequeños pasos entre categorías equivalentes, con un mínimo margen de traspaso de barreras. Las posiciones independientes urbanas juegan un papel clave en este sentido: complementadas con educación universitaria garantizan a los hijos e hijas de estos trabajadores/as el acceso desde los estratos intermedios hacia posiciones dependientes en la clase de servicio. En ausencia de esta conversión de capital económico en capital cultural, otros hijos e hijas enfrentarán como destino probable un descenso hacia ocupaciones asalariadas de bajo rango.

Los datos del 2009 tienden a destacar las barreras a la movilidad que hacen de Chile un país con oportunidades menos abiertas que lo indicado a comienzos de la década. La inferencia que se puede hacer a partir de estos resultados con respecto a relación entre desigualdad de ingresos y movilidad ocupacional apunta en favor de una tesis de dependencia de los recursos: su disponibilidad o ausencia incide en la reproducción de la desigualdad entre generaciones (Bourdieu 1979; Torche 2005). De acuerdo con este enfoque, el control de recursos resulta concomitante a relaciones de dominación, por cuanto quien los posee los utiliza para reproducir su situación de privilegio relativo a quienes están en desventaja. En ausencia de restricciones externas, como políticas públicas orientadas a la redistribución de recursos y oportunidades, el control de recursos se convierte en el factor que explica la transmisión intergeneracional de las desigualdades en una sociedad. El resultado presentado se opone a la interpretación de la fluidez en la movilidad en la desigual sociedad chilena como resultado de la motivación por la alta recompensa a una movilidad exitosa.

Existen consecuencias para la política social derivadas de la estructura de oportunidades detectada en el estudio. El diseño de la política social debiera considerar que las posiciones ocupacionales más bajas están más distantes de la clase media que las posiciones medias entre sí y que las posiciones medias contiguas a la clase más alta también están a gran distancia de ella. En suma, tanto los peldaños de salida de posiciones desfavorecidas, como los de acceso a las más favorables, son los más altos de la jerarquía social. Entre ellos un extendido plano de posiciones equivalentes que componen estratos medios incomparables con la clase media del siglo XX. 


\section{REFERENCIAS BibLIOGRÁFICAS}

Agresti, A. 2002. Categorical Data Analysis. Nueva York: Wiley-Interscience.

Andrews, D. and A. Leigh. 2009. "More inequality, less social mobility". Applied Economics Letters 16:1489-1492.

Araujo, K. y D. Martuccelli. 2011. "La inconsistencia posicional: un nuevo concepto sobre la estratificación social". Revista de la CEPAL 103:165-178.

Atria, Raúl. 2004. "Estructura ocupacional, estructura social y clases sociales". Serie Políticas Sociales 96 (LC/L.2192-P/E). Santiago: Comisión Económica para América Latina y el Caribe (CEPAL).

Björklund, A. and M. Jäntti. 1997. "Intergenerational income mobility in Sweden compared to the United States". American Economic Review 87:1009-1018.

Bourdieu, P. 1979. La distinction. Critique sociale du jugement. París: Éditions de Minuit.

Contreras, D. 2003. "Poverty and inequality in a rapid growth economy: Chile 1990-96". Journal of Development Studies 39:181-200.

Corak, M. 2006. "Do poor children become poor adults? Lessons from a cross country comparison of generational earnings mobility". Research on Economic Inequality 13:143-188.

Duncan, Otis D. 1966. "Methodological Issues in the Analysis of Social Mobility". Pp. 51-97 en Social Structure and Mobility in Economic Development, editado por N. J. Smelser \& S. M. Lipset. London: Routledge and Kegan Paul.

Dunn, C. 2007. "The intergenerational transmission of lifetime earnings: Evidence from Brazil". The B. E. Journal of Economic Analysis \& Policy 7(2).

Erikson, R. y J. H. Goldthorpe. 1992. The Constant Flux: a Study of Class Mobility in Industrial Societies. Oxford [England]: Clarendon Press.

Espinoza, V. 2002. "La movilidad ocupacional en el Cono Sur. Acerca de las raíces estructurales de la desigualdad social”. Proposiciones 34:31-40.

Espinoza, V. 2006. "La movilidad ocupacional en el Cono Sur. Oportunidades y desigualdad social". Revista de Sociología Universidad de Chile 2:131-146.

Espinoza, V. y E. Barozet. 2009. "De qué hablamos cuando decimos "clase media"? Perspectivas sobre el caso chileno". Pp. 103-130 en El Arte de Clasificar a los Chilenos, editado por Alfredo Joignant \& Pedro Güell. Santiago: Ediciones Universidad Diego Portales.

Filgueira, C. 2001. "La actualidad de viejas temáticas: sobre los estudios de clase, estratificación y movilidad social en América Latina". Serie Políticas Sociales 51 (LC/L.1582-P). CEPAL. Comisión Económica para América Latina y el Caribe. Santiago.

Fox, J. 2008. Applied Regression Analysis and Generalized Linear Models. Thousand Oaks (CA): SAGE Publications, Inc.

Ganzeboom, Harry B. G. y Donald J. Treiman. 1996. "Internationally Comparable Measures of Occupational Status for the 1988 International Standard Classification of Occupations". Social Science Research 25:201-239. 
Goldthorpe, J. y A. McKnight. 2006. "The Economic Basis of Social Class". Pp. 109-136 en Mobility and Inequality. Frontiers of Research in Sociology and Economics, editado por Stephen L. Morgan, David B. Grusky \& Gary S. Fields. Stanford: Stanford University Press.

Goodman, L. A. 1985. "The Analysis of Cross-Classified Data Having Ordered and/or Unordered Categories: Association Models, Correlation Models and Asymmetry Models for Contingency Tables With or Without Missing Entries". The Annals of Statistics 13:10-69.

Grusky, David B. y Jesper B. Sørensen. 1998. "Can Class Analysis be Salvaged?”. American Journal of Sociology 1035:1187.

Gurrieri, A. y P. Sáinz. 2003. "Empleo y movilidad estructural. Trayectoria de un tema prebischiano". Revista de la CEPAL 80:141-164.

Hout, M. 1988. "More universalism, less structural mobility: The American occupational structure in the 1980's". American Journal of Sociology 93:1358-1400.

Hout, M. y Thomas A. DiPrete. 2006. "What we have learned: RC28's contributions to knowledge about social stratification". Research in Social Stratification and Mobility 24:1-20.

Jorrat, Jorge R. 2000. Estratificación social y movilidad. Un estudio del Área Metropolitana de Buenos Aires. Serie Estudios sobre ciencia, tecnología y sociedad. Tucumán: Universidad Nacional de Tucuman, Secretaria de Ciencia y Técnica.

Kay, C. y P. Silva. 1992. Development and Social Change in the Chilean Countryside: From the PreLand Reform Period to the Democratic Transition. Amsterdam: CEDLA

Kessler, G. y V. Espinoza. 2007. "Movilidad social y trayectorias ocupacionales en Buenos Aires. Continuidades, rupturas y paradojas". Pp. 259-301 en Estratificación y movilidad social en América Latina, coordinado por Rolando Franco, Arturo León, \& Raúl Atria. Santiago de Chile: LOM Ediciones.

Kuha, J. y D. Firth. 2011. "On the index of dissimilarity for lack of fit in loglinear and log-multiplicative models". Computational Statistics \& Data Analysis 55:375-388.

Leiulfsrud, Håkon, Ivano Bison \& Heidi Jensberg. 2005. "Social class in Europe. European Social Survey 2002/3." NTNU Samfunnsforskning/NTNU Social Research Ltd. Trondheim

Ministerio de Educación de Chile (Mineduc). 2009. Indicadores de la Educación en Chile 2007-2008. Santiago de Chile: Ministerio de Educación.

Naciones Unidas. Programa de las Naciones Unidas para el Desarrollo. Informe de Desarrollo Humano Chile Santiago. 2010. Desarrollo humano en Chile 2010. Género: Ios desafíos de la igualdad. Chile: PNUD.

Núñez, J. y A. Tartakowsky. 2011. "The relationship between income inequality and inequality of opportunity in a high-inequality country: the case of Chile". Applied Economics Letters 184:359-369.

Núñez, J. I. y L. Miranda. 2010. "Intergenerational Income Mobility in a Less-Developed, High-Inequality Context: The Case of Chile". The B. E. Journal of Economic Analysis \& Policy 10.

Núñez J. y L. Miranda. 2010, "Intergenerational income and educational mobility in urban Chile". Estudios de Economía 38:195-221.

R Development Core Team. 2010. R: A Language and Environment for Statistical Computing. Vienna:

R Foundation for Statistical Computing. 
Solon, G. 2002. "Cross-country differences in intergenerational earnings mobility". Journal of Economic Perspectives 16:59-66.

Solon, G. 2004. "A model of intergenerational mobility variation over time and place". Pp.38-47 in Generational Income Mobility in North America and Europe, editado por Miles Corak. Cambridge: Cambridge University Press.

Solís, P. 2007. Inequidad y movilidad social en Monterrey. México D.F.: El Colegio de México.

Torche, F. 2005. "Unequal but fluid: social mobility in Chile in comparative perspective". American Sociological Review 70:422-450.

Torche, F. y G. Wormald. 2004. "Estratificación y movilidad social en Chile: entre la adscripción y el logro". Serie Políticas Sociales 98 (LC/L.2209-P). Santiago: Comisión Económica para América Latina y el Caribe.

Treiman, D. J. 2009. Quantitative Data Analysis: Doing Social Research to Test Ideas. Research Methods for the Social Sciences San Francisco: Jossey-Bass/Wiley

Turner, H. y D. Firth. 2010. "Generalized nonlinear models in R: An overview of the gnm package." R package version 1.0-0. R package version 1.0-1 (http://CRAN.R-project.org/package=gnm).

Zimmerman, D. J. 1992. "Regression toward mediocrity in economic stature". The American Economic Review 82:409-429.

Vicente Espinoza es Profesor Asociado del Instituto de Estudios Avanzados (IDEA) de la Universidad de Santiago. Investigador Asociado del Centro de Estudios del Conflicto y la Cohesión Social (FONDAP-CONICYT). Titular de la Cátedra Andrés Bello 2011 en la Universidad de Leiden. Sus áreas de investigación incluyen desigualdad social, movilidad ocupacional y redes sociales. Autor de libros y artículos en revistas académicas nacionales y extranjeras.

Javier NúÑEZ es Profesor Asociado del Departamento de Economía de la Universidad de Chile. Investigador Asociado del Centro de Estudios del Conflicto y la Cohesión Social (FONDAP-CONICYT). Fue Director del Departamento de Economía (2006 y 2008). Sus áreas de investigación incluyen la aplicación de la Teoría de Juegos a la regulación económica, movilidad social y discriminación laboral. Es autor de varios artículos en revistas académicas nacionales y extranjeras y capítulos de libros. 


\section{ANEXo}

Tabla de movilidad ocupacional intergeneracional en Chile. Porcentaje del "flujo de salida"

\begin{tabular}{lccccccccc}
\hline $\begin{array}{l}\text { Ocupación del padre o } \\
\text { sostenedor del entrevistado } \\
\text { (origen) }\end{array}$ & \multicolumn{10}{c}{ Ocupación del entrevistado (destino) } & & N=100\% \\
\hline & I+II & III & IVa+b & IVc & V+VI & VIla & VIllb & \\
\hline I+II. Clase de servicios & 61,3 & 10,9 & 13,9 & 0,8 & 5,0 & 6,7 & 1,3 & 238 \\
III. Rutina no manual & 26,6 & 27,6 & 12,5 & 0 & 15,1 & 18,2 & 0 & 192 \\
IVa+b. Peq. empresario & 27,8 & 19,1 & 23,0 & 1,0 & 15,6 & 12,4 & 1,2 & 418 \\
IVc. Peq. agricultor & 10,0 & 7,2 & 17,9 & 17,9 & 8,4 & 24,7 & 13,9 & 251 \\
V+VI. Trab. calificado & 21,1 & 12 & 19,2 & 1,2 & 17,6 & 25,9 & 3,0 & 432 \\
VIlla. Trab. no calificado & 16,5 & 17,9 & 17,1 & 1,4 & 12,8 & 31,7 & 2,6 & 665 \\
VIIb. Campesino & 5,4 & 10,1 & 11,6 & 12,2 & 11,3 & 25,4 & 23,9 & 335 \\
Total & 22,0 & 15,1 & 17,1 & 4,2 & 12,9 & 22,6 & 6,0 & 2531 \\
\hline
\end{tabular}

Fuente: Encuesta ENES 2009.

Clave de lectura.

Los porcentajes representan el destino de entrevistados/as con un mismo origen social, indicado por la posición de sus padres o los proveedores principales de sus hogares cuando ellos tenían 15 años de edad. 